Signal \& Image Processing : An International Journal (SIPIJ) Vol.5, No.3, June 2014

\title{
GLOBAL THRESHOLD AND REGION-BASED ACTIVE CONTOUR MODEL FOR ACCURATE IMAGE SEGMENTATION
}

\author{
Nuseiba M. Altarawneh ${ }^{1}$, Suhuai Luo ${ }^{1}$, Brian Regan ${ }^{1}$, Changming Sun ${ }^{2}$, \\ Fucang $\mathrm{Jia}^{3}$ \\ ${ }^{1}$ School of Design Communication and IT, the University of Newcastle, \\ Callaghan NSW 2308, Australia \\ ${ }^{2}$ CSIRO Computational Informatics, Locked Bag 17, \\ North Ryde, NSW 1670, Australia \\ ${ }^{3}$ Shenzhen Institutes of Advanced Technology, \\ Chinese Academy of Sciences, Shenzhen 518055, China
}

\begin{abstract}
In this contribution, we develop a novel global threshold-based active contour model. This model deploys a new edge-stopping function to control the direction of the evolution and to stop the evolving contour at weak or blurred edges. An implementation of the model requires the use of selective binary and Gaussian filtering regularized level set (SBGFRLS) method. The method uses either a selective local or global segmentation property. It penalizes the level set function to force it to become a binary function. This procedure is followed by using a regularisation Gaussian. The Gaussian filters smooth the level set function and stabilises the evolution process. One of the merits of our proposed model stems from the ability to initialise the contour anywhere inside the image to extract object boundaries. The proposed method is found to perform well, notably when the intensities inside and outside the object are homogenous. Our method is applied with satisfactory results on various types of images, including synthetic, medical and Arabic-characters images.
\end{abstract}

\section{KEYWORDS}

Active contour model, Level set method, CV model, ZAC model.

\section{INTRODUCTION}

Active contour model (ACM) signifies one of the most successful techniques in dealing with image segmentation problems. The idea behind the ACM is to evolve a curve or a surface defined within an image from some arbitrary initial shape towards its interior normal direction and stop it on the object boundary [1]. The parametric curve is linked with an energy function. During the deformation, the curve tries to minimize its energy so that the final curve possesses a local minimum when the contour is spatially aligned with the shape or the desired image features. Thus the problem of segmentation is reduced to an energy minimization problem [2].

In order to locate the desired image features, parametric curves are initialized close to the desired feature and are forced to move toward the local minimum that is located on the desired features under the influence of internal and external forces. The internal forces are defined within the curve or surface to keep the model smooth during the deformation. The external DOI : 10.5121/sipij.2014.5301 
forces are derived from the image data to move the curve toward the object boundary or the desired features within an image.

Owing to its flexibility in allowing topological changes, the level set method has been extensively utilized in problems such as curve evolution, especially the curve motion by mean curvature as described by Osher and Sethian [3]. In the level set method, the evolution curve is represented implicitly via a Lipchitz function $\phi$, as $C=\{(x, y) \mid \phi(x, y)=0\}$. The zero level set of the function $\phi(t, x, y)$ represents the evolution curve $C$ at time $t$. The evolution of the curve $C$ in a normal direction with speed $F$ is obtained by solving the equation:

$\left\{\begin{array}{l}\frac{\partial \phi}{\partial t}=|\nabla \phi| F \quad \text { in }[0, \infty] \in R^{2} \\ \phi(x, y, 0)=\phi_{0}(x, y) \text { in } R^{2}\end{array}\right.$

where $\{(x, y) \mid \phi(x, y)=0\}$ represents the initial contour. The geodesic active contour models $[4,5]$ utilize the image gradient in order to construct an edge detector function. The objective of this function is to stop the contour evolution on the object boundary. The general edge detector function can be defined by a positive and decreasing function such as:

$$
g\left(\left|\nabla u_{0}\right|\right)=\frac{1}{1+\left|\nabla G_{\sigma}(x, y) * u_{0}(x, y)\right|}
$$

where $u_{0}$ is a given image in $\Omega$ and $G_{\sigma} * u_{0}$ denotes a smooth version of $u_{0}$ after convolving it with the Gaussian function. The values of $g\left(\left|\nabla u_{0}\right|\right)$ function will be positive in the homogenous regions and zero on the object boundary. A particular case is the motion by mean curvature in which $F=\operatorname{div}(\nabla \phi(x, y) /|\nabla \phi(x, y)|)$. Malladi et al. [6] proposed the following level set equation:

$$
\left\{\begin{array}{l}
\frac{\partial \phi}{\partial t}=|\nabla \phi|\left(-v+\frac{v}{\left(m_{1}+m_{2}\right)}\left(\left|\nabla G_{\sigma} * u_{0}\right|-m_{2}\right)\right) \\
\phi(x, y, 0)=\phi_{0}(x, y) \text { in } R^{2}
\end{array}\right.
$$

where $v$ is a constant, and $m_{1}$ and $m_{2}$ are the maximum and minimum values of the image gradient $\left|\nabla G_{\sigma} * u_{0}\right|$, respectively. The evolving curve stops at the boundary, i.e., points with the highest gradient. Caselles et al. [5] proposed a Geometric Active Contour model (GAC) based on the mean curvature motion:

$\left\{\begin{array}{l}\frac{\partial \phi}{\partial t}=g\left(\left|\nabla u_{0}\right|\right)|\nabla \phi|\left(\operatorname{div}\left(\frac{\nabla \phi}{|\nabla \phi|}\right)+v\right) \quad \text { in }[0, \infty] \in R^{2} \\ \phi(x, y, 0)=\phi_{0}(x, y) \text { in } R^{2}\end{array}\right.$

where $v$ is a constant. In GAC the curve moves in the normal direction with a speed equal to $g\left(\left|\nabla u_{\circ}\right|\right)(\operatorname{div}(\nabla \phi|| \nabla \phi \mid)+v)$. The curve will stop the evolution when the function $g$ vanishes.

All the above ACMs are termed as edge-based models [7-10] because they utilize the image gradient as stopping criterion for the evolving curve. Edge-based models do not perform well in the presence of noise and in images with weak edges or without edges. In the case of a discrete gradient, the curve may pass through the edges because the function $g\left(\left|\nabla u_{0}\right|\right)$ never 
approaches zero at these points. These models possess a local segmentation property. They are sensitive to the position of the initial contour as they are prone to the local minima and can only segment the desired object with a proper initial contour. As a result, these models fail to detect the boundaries when the initial contour is far from the boundary of the desired object. They also cannot detect the interior boundary without setting a proper initial contour inside the desired object.

Region-based models represent another category of ACMs [11-16]. These models deploy statistical information inside and outside the contour in order to control its evolution. Regionbased models are less sensitive to the position of the initial contour. They perform well in the presence of noise and on images with weak edges or without edges. These models have a global segmentation property and can detect the interior and exterior boundaries at the same time, regardless of the position of the initial contour in the image. Chan and Vese [11] proposed a widely used region-based model, namely the CV model. Zhang et al. [12] proposed a ZAC model which uses statistical information inside and outside the contour to formulate the signed pressure force when evolving the contour. This paper proposes an improved ZAC model which can perform well when the edge is weak or blurred.

This paper is organized as follows: Section 2 reviews the CV model [11]. Section 3 reviews the ZAC model [12]. Section 4 describes our methodology. Section 5 shows some experimental results and finally the conclusion is made in Section 6.

\section{THE CV MODEL}

The CV model is based on the Mumford-Shah segmentation technique [13]. It has been successfully implemented in binary phase segmentation. The CV model uses the statistical information inside and outside the contour with the aim of controlling the evolution. The CV model is formulated by minimizing the equation:

$$
F\left(c_{1}, c_{2}, C\right)=\mu \text { Length }(C)+v \text { Area }(\text { inside }(C))+\lambda_{1} \int_{\text {inside }}(C)\left|u_{0}(x, y)-c_{1}\right|^{2} d x d y+\lambda_{2} \int_{\text {outside }(C)}\left|u_{0}(x, y)-c_{2}\right|^{2} d x d y
$$

where $u_{0}$ is a given image in $\Omega, \mu, v, \lambda_{1}, \lambda_{2}$ are positive parameters and $c_{1}, c_{2}$ are the average intensities inside and outside the curve $C$, respectively. With the level set method, one can assume:

$\left\{\begin{array}{l}C=\{(x, y) \in \Omega: \phi(x, y)=0\} \\ c_{1}=\{(x, y) \in \Omega: \phi(x, y)>0\} \\ c_{2}=\{(x, y) \in \Omega: \phi(x, y)<0\}\end{array}\right.$
$\left\{\begin{array}{l}c_{1}(\phi)=\frac{\int_{\Omega} u_{0}(x, y) H(\phi(x, y)) d x d y}{\int_{\Omega} H(\phi(x, y)) d x d y} \\ c_{2}(\phi)=\frac{\int_{\Omega} u_{0}(x, y)(1-H(\phi(x, y))) d x d y}{\int_{\Omega}(1-H(\phi(x, y))) d x d y}\end{array}\right.$ 
where $H(\phi)$ refers to the Heaviside function and $\delta(\phi)$ is the Dirac function. The regularization version of $H$ and $\delta$ that were implemented in the $\mathrm{C}-\mathrm{V}$ model are:

$$
\left\{\begin{array}{l}
H_{\varepsilon}(z)=\frac{1}{2}\left(1+\frac{2}{\pi} \arctan \left(\frac{z}{\varepsilon}\right)\right) \\
\delta(z)=\frac{1}{\pi} \cdot \frac{\varepsilon}{\varepsilon+z}, \quad z \in R
\end{array}\right.
$$

The corresponding variation level set formulation is then:

$$
\frac{\partial \phi}{\partial t}=\delta(\phi)\left[\mu \operatorname{div}\left(\frac{\nabla \phi}{|\nabla \phi|}\right)|\nabla \phi|-v-\lambda_{1}\left(u_{0}-c_{1}\right)^{2}+\lambda_{2}\left(u_{0}-c_{2}\right)^{2}\right]
$$

where $\mu$ controls the curve smoothness during the deformation, $v$ is a constant to increase the propagation speed, and $\lambda_{1}$ and $\lambda_{2}$ control the image forces inside and outside the contour $C$, respectively. The values of $\delta_{\varepsilon}(z)$ tend to be near zero, if $\varepsilon$ is too small. In this case, extraction of the desired object may fail if the initial contour starts far from the desired object. The final contour location may not be accurate if $\mathcal{E}$ is large [12].

\section{THE ZAC MODEL}

Zhang et al. [12] proposed a novel level set method termed as selective binary and Gaussian filtering regularized level set (SBGFRLS). This approach selectively penalizes the level set function to be a binary function. This is followed by using a Gaussian function to regularize it. The Gaussian filters smooth the level set function and afford the evolution more stability. SBGFRLS model reduces the computational cost of the re-initialization step which in turn makes it more efficient than the traditional level set methods [17].

It is worthwhile mentioning that the SBGFRLS method has the advantage of being a general and robust technique. It can be applied to the classical ACMs, such as the GAC model [4] as well as the CV model [11].

A novel signed pressure force (SPF) [18] is proposed to control the direction of the evolution and to stop the evolving contour at weak or blurred edges. Zhang et al.'s model is referred as Zhang et al. active contour (ZAC). The ZAC model uses statistical information inside and outside the contour to formulate the SPF. The proposed SPF function is assigned with values in the range $[-1,1]$. It modulates the signs of the pressure forces inside and outside the region of interest as:

$$
\operatorname{spf}\left(u_{0}(x)\right)=\frac{u_{0}(x)-\frac{c_{1}+c_{2}}{2}}{\max \left(\left|u_{0}(x)-\frac{c_{1}+c_{2}}{2}\right|\right)}, \quad x \in \Omega
$$

where $c_{1}$ and $c_{2}$ are defined in Eq. (6). The SPF assumes that if the intensities inside and outside the object are homogenous, it is instinctive that $\operatorname{Min}\left(u_{0}(x)\right)<c_{1}$ and $\operatorname{Max}\left(u_{0}(x)\right)<c_{2}$, therefore $\operatorname{Min}\left(u_{o}(x)\right)<\frac{c_{1}+c_{2}}{2}<\operatorname{Max}\left(u_{o}(x)\right)$. The SPF function has opposite signs around the object boundary in order to force the contour to shrink when it is outside the object and to expand when it is inside the object. 
The formulation of the level set function in the ZAC model is given as:

$$
\frac{\partial \phi}{\partial t}=\operatorname{spf}\left(u_{0}(x)\right) \cdot \alpha|\nabla \phi|, \quad x \in \Omega
$$

The ZAC model utilizes a binary function for the initialization of the level set function $\phi$ instead of using the signed distance function as in the traditional level set method. The ZAC model deploys the image statistical information to stop the curve evolution on the desired object boundaries. This makes the ZAC model insensitive to noise and can perform well in the case of an object with weak edges or without edges. The ZAC model is capable of performing both local and global segmentation, in contrast to the CV model which can only handle global segmentation and extracts all the objects. The ZAC model has less computational complexity than the GAC and CV models.

\section{THE PROPOSED MODEL}

In this paper, we propose a new edge stopping function that controls the direction of the evolution and stops the evolving contour at weak or blurred edges. Our model is implemented using the SBGFRLS method, which grants it a selective local or global segmentation property. Our model mainly adapts the methodology of the ZAC model [12] yet with improvement. Our novel modification stems from utilizing a new function termed the global threshold function (GTF) instead of using the SPF as in the original ZAC model. The GTF operates similarly to the SPF. Generally, both functions produce similar results. It controls the direction of the evolution and stops the evolving contour at weak or blurred edges.

Our proposed model performs well when the intensities inside and outside the object are homogenous and in the binary segmentation phase, in an analogy to the ZAC model. The GTF has opposite signs around the object boundary in order to force the contour to shrink when it is outside the object and to expand when it is inside the object. The proposed GTF is assigned with values in the range $[-1,1]$ as:

$$
g t f\left(u_{0}(x)\right)=\frac{u_{0}(x)-t}{\max \left(\left|u_{0}(x)-t\right|\right)}, x \in \Omega
$$

where $t$ is a threshold value computed automatically by using the global thresholding method [19]. For choosing $t$ automatically, the following algorithm is applied:

1. Select an initial estimate for $t$. (A suggested initial value is the midpoint between the minimum and maximum intensity values in the image.)

2. Segment the image using $t$. This will produce two groups of pixels, G1 consisting of all pixels with intensity values $>t$, and $\mathrm{G} 2$, consisting of pixels with values $<=t$.

3. Compute the average intensity values $x_{1}$ and $x_{2}$ for the pixels in regions G1 and G2.

4. Compute a new threshold value: $t=1 / 2\left(x_{1}+x_{2}\right)$

5. Repeat steps 2 through 4 until the difference in $t$ in successive iterations is smaller than a predefined parameter $t_{0}$.

The procedure of the proposed algorithm consists of consecutive steps. Firstly, it requires initialising the level set function into a binary function and computing the value of $t$ and the value of stopping function according to Eq. (11). In the next step, Eq. (10) is deployed to evolve the level set function. In this procedure, if the local segmentation property is desired, let $\phi=1$ if $\phi$ 
$>0$; otherwise $\phi=-1$. A Gaussian filter is to be used to smooth and regularize the level set function. Finally, the procedure stops upon the convergence of the evolution of the level set function. The contour could be initialized anywhere inside the image to extract all exterior and interior boundaries, even if the initial contour does not surround all the objects in the image. Our model entails all the advantages pertinent to the ZAC model. Our model gives a similar result to the ZAC model in less computational time because the values of $t$ and $\operatorname{gtf}\left(u_{0}(x)\right)$ are computed only once. The ZAC model can extract objects with distinctive boundaries while interior intensities are not homogeneous. By contrast, our model extracts both the interior and exterior boundaries as shown in Figure 6.

\section{EXPERIMENTAL RESULTS}

In each experiment, we selected values of $\rho, \mathcal{E}, \sigma, k$, and $s$ to be $1,1.5,1,5$ and 1 , respectively. The values of $\alpha$ and $t$ were set according to the images. Figure 1 exhibits the global segmentation property of the proposed model. The initial contour is initialized far from the objects, as shown in the first row of Figure 1. The second row shows the segmentation results of our model. Clearly, our model extracts accurately all the objects in the image regardless of the position of the initial contour.
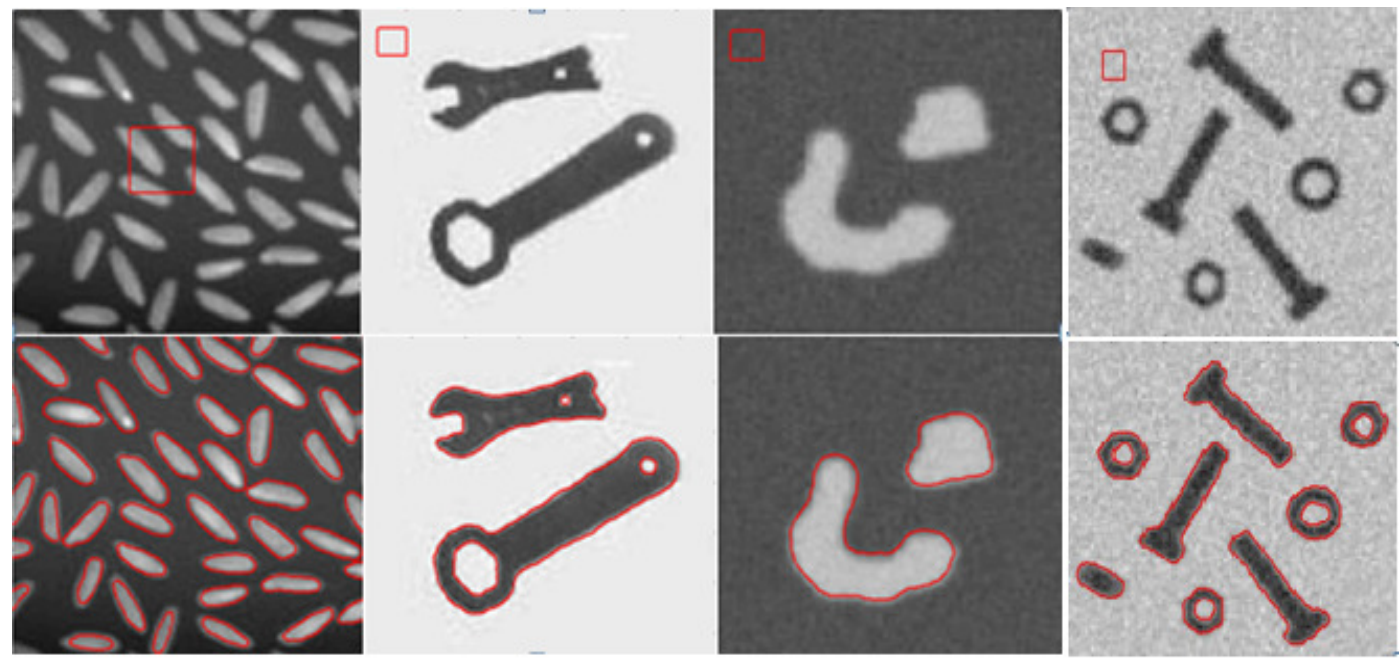

Figure 1. The first row shows the initial contour; the second row shows the segmentation results of the proposed method with $t$ corresponding to $104,150,132$ and 138 for the first, second, third and fourth column, respectively and $\alpha=20$.

Figure 2 exhibits the global segmentation property of the CV model and our proposed model. The initial contour is initialized far from the objects, as shown in the first image of Figure 2. The middle image shows the segmentation results of the CV model. As is displayed, the CV model fails to extract all the objects in the image. The third image shows the segmentation result of our model. Clearly, our model extracts accurately all the objects in the image regardless of the position of the initial contour, while the $\mathrm{CV}$ model may be trapped into the local minima resulting in unsatisfactory segmentation. 

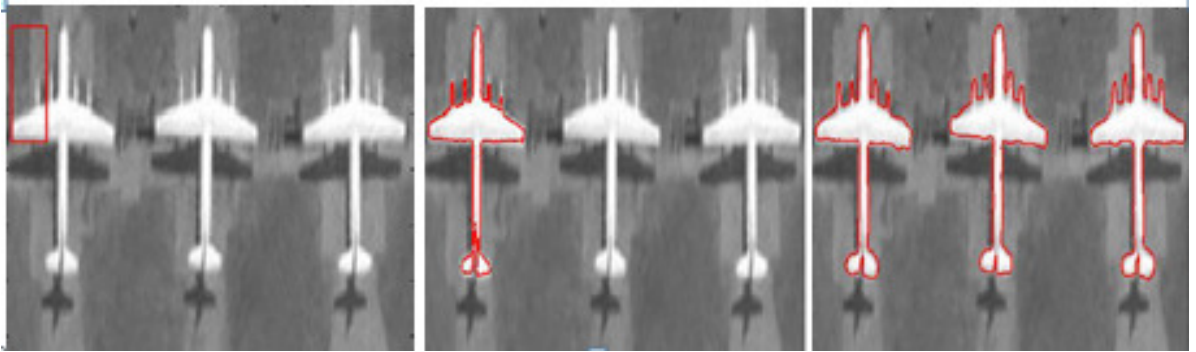

Figure 2. Comparisons of the global property between the CV model and the proposed method. The first image shows the initial contour; the middle image shows the segmentation results of the CV method; and the third image shows the result of the proposed method with $t=172$ and $\alpha=20$.

Figure 3 presents segmentation results of the $\mathrm{CV}$ model and the proposed model in a magnetic resonance image of the left ventricle of a human heart. In an analogy to the ZAC model, our model also can selectively extract the desired object by setting the initial contour inside or surrounding the desired boundaries, while the CV model will extract all the objects. Furthermore, the evolution direction in our model can be controlled to obtain satisfactory segmentation results, while the $\mathrm{CV}$ model may obtain disordered results.
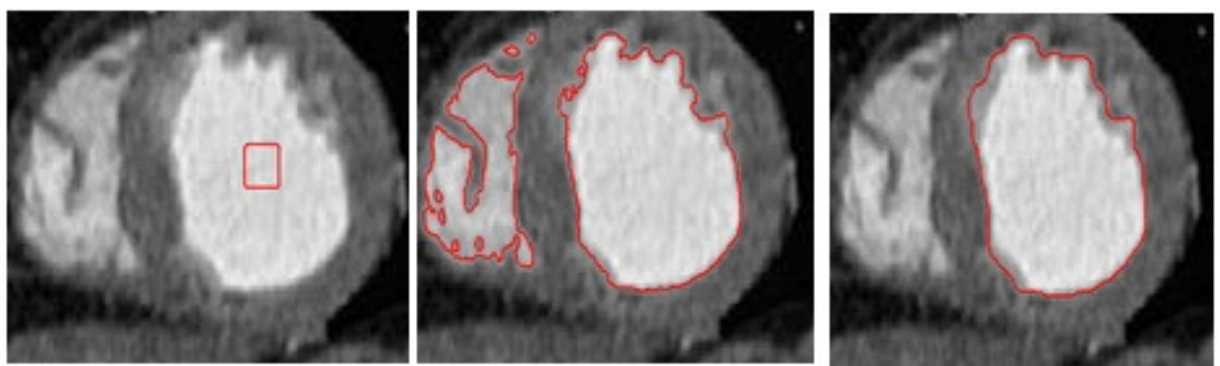

Figure 3. Segmentation results for a magnetic resonance image of the left ventricle of a human heart. The first image shows the initial contour; the middle image shows the segmentation results of the $\mathrm{CV}$ model; and the third image shows the result of the proposed model with $t=111$ and $\alpha=5$.

Figure 4 shows the local segmentation property of the proposed model. The initial contour resides near or surrounding the desired objects, as shown in the first row of Figure 4. The second row shows the segmentation results of our proposed model. 

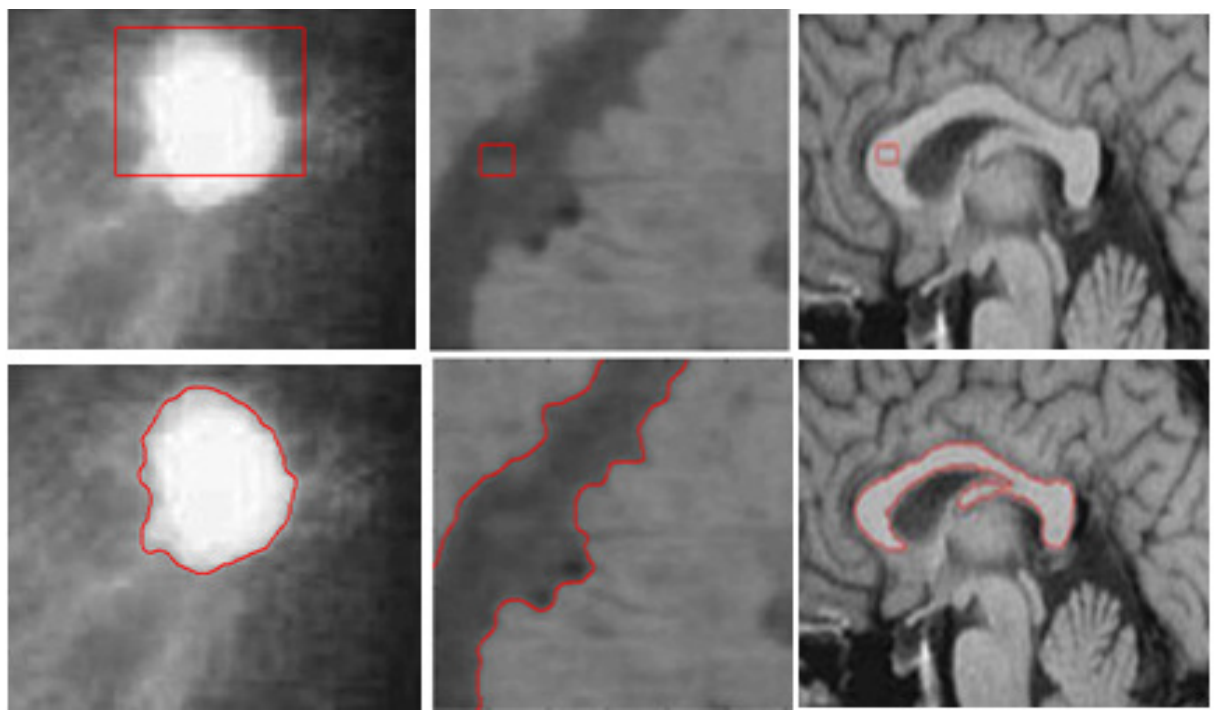

Figure 4. Segmentation results of the proposed model. The first row shows the initial contours; the second row shows the segmentation results of the proposed method. $t$ equals to $157,155,138$ for the first, second and third column, respectively and $\alpha=20$.

Figure 5 shows the global segmentation results by the proposed model for noisy images. As it can be seen, despite of the presence of significant noise inherit in the image, our model performs well in detecting the desired object boundary.
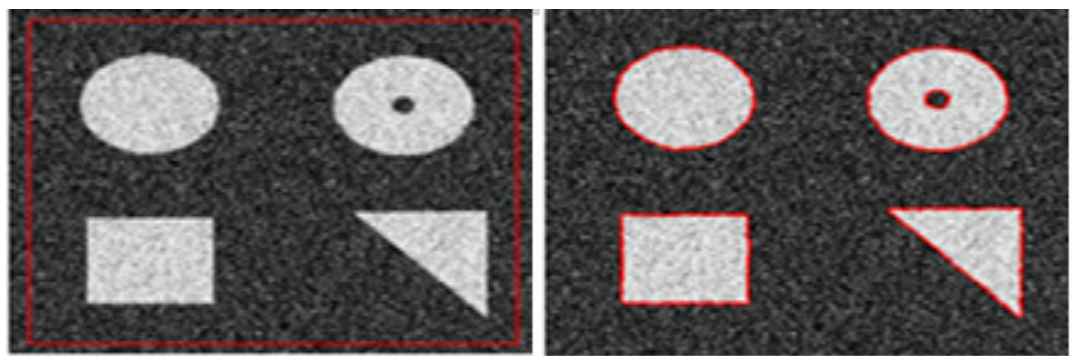

Figure 5. Global segmentation results for a noisy image. The left image shows the initial contour; the right image shows the segmentation result of the proposed method with $t=125$ and $\alpha=10$.

Figure 6 shows the local segmentation property of the ZAC model and the proposed model. As shown in Figure 6, the ZAC model extracts objects with distinct boundaries whereas the interior intensities are not homogeneous. On the contrary, our model extracts the interior and the exterior boundaries. This represents the main shortcoming of our propose model. 
Signal \& Image Processing : An International Journal (SIPIJ) Vol.5, No.3, June 2014
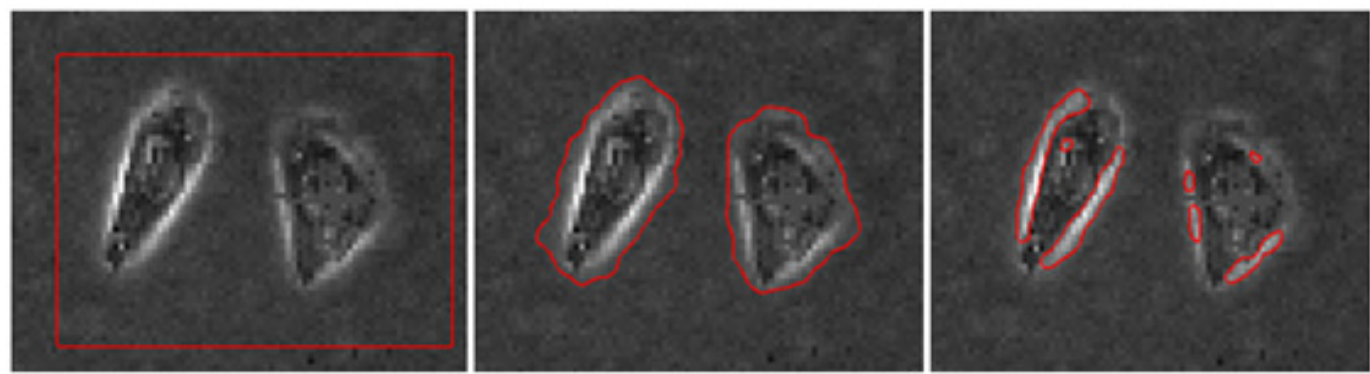

Figure 6. Local segmentation results for a real microscope cell image. The first image shows the initial contour; the middle image shows the segmentation results of the ZAC method; and the third image shows the result of the proposed method with $t=94$. The original image is sourced from Zhang et al. [12].

Figure 7 exhibits the performance of our proposed method in the case of Arabic-characters segmentation. As shown in this figure, our proposed model attains satisfactory segmentation results.
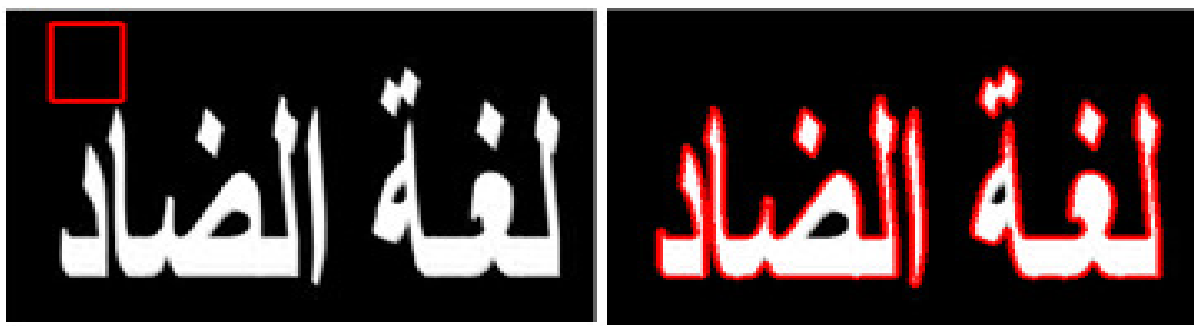

Figure 7. The left image shows the initial contour; the right image shows the segmentation results of the proposed method with $t=120$ and $\alpha=20$.

\section{Conclusions}

A novel global threshold-based active contour model with a new edge-stopping function has been presented. The main merits of this approach consist of its ability to control the direction of the evolving contour and to stop it on the weak or blurred edges. Our model is implemented using the SBGFRLS method. We tested this method on several categories of images including synthetic, medical and Arabic-characters where a satisfactory performance was attained.

\section{REFERENCES}

[1] M. Kass, A. Witkin, and D. Terzopoulos, "Snakes: Active contour models," International Journal of Computer Vision, vol. 1, pp. 321-331, 1988.

[2] C. Xu, D. L. Pham, and J. L. Prince, "Image segmentation using deformable models," Handbook of Medical Imaging, vol. 2, pp. 129-174, 2000.

[3] S. Osher and J. A. Sethian, "Fronts propagating with curvature-dependent speed: algorithms based on Hamilton-Jacobi formulations," Journal of Computational Physics, vol. 79, pp. 12-49, 1988.

[4] V. Caselles, R. Kimmel, and G. Sapiro, "Geodesic active contours," International Journal of Computer Vision, vol. 22, pp. 61-79, 1997.

[5] V. Caselles, F. Catté, T. Coll, and F. Dibos, "A geometric model for active contours in image processing," Numerische Mathematik, vol. 66, pp. 1-31, 1993. 
Signal \& Image Processing : An International Journal (SIPIJ) Vol.5, No.3, June 2014

[6] R. Malladi, J. A. Sethian, and B. C. Vemuri, "Shape modeling with front propagation: A level set approach," IEEE Transactions on Pattern Analysis and Machine Intelligence, vol. 17, pp. 158-175, 1995.

[7] G. Zhu, S. Zhang, Q. Zeng, and C. Wang, "Boundary-based image segmentation using binary level set method," Optical Engineering, vol. 46, pp. 050501-050501-3, 2007.

[8] C. Li, C. Xu, C. Gui, and M. D. Fox, "Level set evolution without re-initialization: a new variational formulation," in IEEE Computer Society Conference on Computer Vision and Pattern Recognition, 2005, pp. 430-436.

[9] N. Paragios and R. Deriche, "Geodesic active contours and level sets for the detection and tracking of moving objects," IEEE Transactions on Pattern Analysis and Machine Intelligence, vol. 22, pp. 266$280,2000$.

[10] C. Xu and J. L. Prince, "Snakes, shapes, and gradient vector flow," IEEE Transactions on Image Processing, vol. 7, pp. 359-369, 1998.

[11] T. F. Chan and L. A. Vese, "Active contours without edges," IEEE Transactions on Image Processing, vol. 10, pp. 266-277, 2001.

[12] K. Zhang, L. Zhang, H. Song, and W. Zhou, "Active contours with selective local or global segmentation: A new formulation and level set method," Image and Vision Computing, vol. 28, pp. 668-676, 2010.

[13] D. Mumford and J. Shah, "Optimal approximations by piecewise smooth functions and associated variational problems," Communications on Pure and Applied Mathematics, vol. 42, pp. 577-685, 1989.

[14] C. Li, C.-Y. Kao, J. C. Gore, and Z. Ding, "Implicit active contours driven by local binary fitting energy," in IEEE Conference on Computer Vision and Pattern Recognition, 2007, pp. 1-7.

[15] L. A. Vese and T. F. Chan, "A multiphase level set framework for image segmentation using the Mumford and Shah model," International Journal of Computer Vision, vol. 50, pp. 271-293, 2002.

[16] R. Ronfard, "Region-based strategies for active contour models," International Journal of Computer Vision, vol. 13, pp. 229-251, 1994.

[17] S. Osher and R. Fedkiw, Level Set Methods and Dynamic Implicit Surfaces vol. 153: Springer, 2003.

[18] C. Xu, A. Yezzi Jr, and J. L. Prince, "On the relationship between parametric and geometric active contours," in Conference Record of the Thirty-Fourth Asilomar Conference on Signals, Systems and Computers, 2000, pp. 483-489.

[19] R. C. Gonzalez and E. Richard, Digital Image Processing: Prentice Hall Press, 2002.

\section{AUTHORS}

Nuseiba Altarawneh is a $\mathrm{PhD}$ student at the University of Newcastle, Australia. Her research interests include computer vision, image analysis, and pattern recognition. She obtained M.E. degrees in Computer science from the University of Jordan in 2009.

Dr. Suhuai Luo received $\mathrm{PhD}$ degree in Electrical Engineering from the University of Sydney Australia in 1995. From 1995 to 2004, he worked as a senior research scientist with the Commonwealth Scientific and Industrial Research Organization Australia and the Bioinformatics Institute Singapore. He is currently a senior lecturer with the University of Newcastle Australia. His research interest is in information technology and multimedia, including health informatics, machine learning, image processing, computer vision, and Internet-oriented IT applications.

Dr Brian Regan is a senior lecturer in IT at the University of Newcastle. He is part of the Applied Informatics Research Group (AIR) with interests in health informatics, visualization and development methodologies.
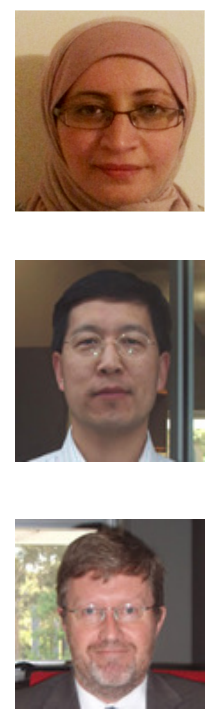
Signal \& Image Processing : An International Journal (SIPIJ) Vol.5, No.3, June 2014

Dr. Changming Sun received the $\mathrm{PhD}$ degree in the area of computer vision from Imperial College London in 1992. Then, he joined CSIRO Computational Informatics, Australia, where he is currently a principal research scientist carrying out research and working on applied projects. His research interests include computer vision, image analysis, and pattern recognition. He has served on the program/organizing committees of various international conferences. He is an Associate Editor for EURASIP Journal on Image and Video Processing, a SpringerOne journal.

Dr. Fucang Jia received PhD degreee in computer application technology from Institute of Computing Technology, Chinese Academy of Sciences in 2004. In 2004-2008, he worked as a research engineer in Shenzhen Anke High-Tech Co., Ltd. From 2008, he joined Shenzhen Institutes of Advanced Technology, Chinese Academy of Sciences as an associate research fellow. His research interests are in computer assisted surgery and machine learning.
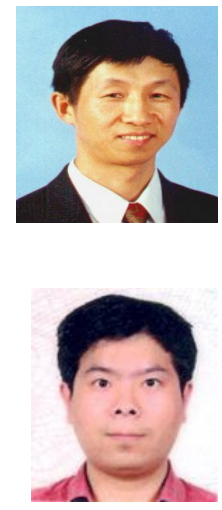